\title{
The development of thermal lattice Boltzmann models in incompressible limit
}

\author{
Nor Azwadi Che Sidik* \\ Department of Thermo-fluid, Faculty of Mechanical Engineering, Universiti Teknologi Malaysia, 81310 UTM Skudai, \\ Johor, Malaysia \\ ${ }^{*}$ To whom correspondence should be addressed. E-mail: azwadi@fkm.utm.my
}

Received 20 September 2007

http:// dx.doi.org/10.11113/mjfas.v3n2.28

\begin{abstract}
In this paper, an incompressible two-dimensional (2-D) and three-dimensional (3-D) thermohydrodynamics for the lattice Boltzmann scheme are developed. The basic idea is to solve the velocity field and the temperature field using two different distribution functions. A derivation of the lattice Boltzmann scheme from the continuous Boltzmann equation for 2-D is discussed in detail. By using the same procedure as in the derivation of the discretised density distribution function, we found that new lattice of four-velocity (2-D) and eight-velocity (3-D) models for internal energy density distribution function can be developed where the viscous and compressive heating effects are negligible. These models are validated by the numerical simulation of the 2-D porous plate Couette flow problem where the analytical solution exists and the natural convection flows in a cubic cavity.
\end{abstract}

| Distribution function | Thermal lattice Boltzmann | Porous Couette flow | Lid-driven cavity flow |

\section{Introduction}

The lattice Boltzmann method is considerably as an alternative approach to the well-known finite difference, finite element, and finite volume techniques for solving the Navier-Stokes equations. Lattice Boltzmann method evolved from Lattice Gas Automata [1], simulates fluid flows by tracking the evolution of the single-particle distribution function. Although as a new comer in numerical scheme, the lattice Boltzmann approach has found recent successes in a host of fluid dynamical problems, including flows in porous media [2], magnetohydrodynamics [3], immiscible fluids [4], and turbulence [5]. However, the simulation of flows with heat transfer turned out to be much more difficult.

Currently, a few thermal lattice Boltzmann models have been proposed. The earliest model which is known as multispeed model [6], uses the same distribution function in defining the macroscopic temperature. However, this model is reported to suffer numerical instability [7] and has a demerit that it can simulate thermal fluid flows only at fixed Prandtl number [8]. As an alternative approach, Shan proposed the so-called passive-scalar model [9]. This model applies that the flow fields (velocity and density) and the temperature are represented by two different distribution functions. The macroscopic temperature is assumed to satisfy the same evolution equation as a passive scale, which is advected by the flow velocity but does not affect the flow field. 
The work of Luo and He [10] showed that the isothermal lattice Boltzmann equation can be directly obtained by properly discretizing the continuous Boltzmann equation in both time and space phases. Following the same procedure, He et al. [11] proposed the double-distribution function model, where the thermal lattice Boltzmann evolution equation can be derived by discretizing the continuous Boltzmann equation for the internal energy distribution. It has been shown that this model is simple and applicable to problems with different Prandtl numbers. More importantly, this model requires low order moment and thus provides higher numerical stability than the passive-scalar model.

In this paper, we developed new four- and eight-velocity lattice models of the internal energy density distribution function for incompressible flow where the compression work done by the pressure and viscous heat dissipation can be neglected. The rest of the paper is organized as follows. In Secs. 2 and 3, we show the theory of the internal energy density distribution function and the discritization procedure of continuous Boltzmann equation which will lead in developing of our new type of four- and eight-velocity lattice models. In Sec. 4, we employ our models to simulate the porous plate Couette flow and natural convection in a cubic cavity for two and three dimensional problems respectively. The final section concludes this study.

\section{Theory of Double-Population Thermal Lattice Boltzmann Model}

We start from the derivation of the internal energy density distribution from the continuous Boltzmann equation. The Boltzmann equation with the Bhatnagar-Gross-Krook (BGK), or single-relaxation-time approximation [12] with external force is given by

$$
\frac{\partial f}{\partial t}+\mathbf{c} \frac{\partial f}{\partial \mathbf{x}}=-\frac{1}{\tau_{v}}\left(f-f^{e q}\right)+F_{f}
$$

where $f(\boldsymbol{x}, \boldsymbol{c}, t)$ is the single-particle distribution function, $\boldsymbol{c}$ is the microscopic velocity, $\tau_{v}$ is the relaxation time due to collision, $F_{f}$ is the external force, and $f^{e q}$ is the local Maxwell-Boltzmann equilibrium distribution function given by

$$
f^{e q}=\rho\left(\frac{1}{2 \pi R T}\right)^{D / 2} \exp \left\{-\frac{(\mathbf{c}-\mathbf{u})^{2}}{2 R T}\right\}
$$

where $R$ is the ideal gas constant, $D$ is the dimension of the space, and $\rho, \boldsymbol{u}$, and $T$ are the macroscopic density, velocity, and temperature respectively. The macroscopic variables $\rho, \boldsymbol{u}$, and $T$ can be evaluated as the moment to the distribution function as follow

$$
\rho=\int f d \mathbf{c}, \rho \mathbf{u}=\int \mathbf{c} f d \mathbf{c}, \frac{\rho D R T}{2}=\int \frac{(\mathbf{c}-\mathbf{u})^{2}}{2} f d \mathbf{c}
$$

By applying the Chapmann-Enskog expansion [13], the above equations can lead to macroscopic continuity, momentum and energy equation. However the Prandtl number obtained is fixed to a constant value [14]. This is caused by the use of single relaxation time in the collision process. The relaxation time of energy carried by the particles to its equilibrium is different to that of momentum. Therefore we need to use a different two relaxation times to characterize the momentum and energy transport. This is equivalent in introducing a new distribution function to define energy.

To obtain the new distribution function modeling energy transport, the new variable, the internal energy density distribution function is introduced

$$
g=\frac{(\mathbf{c}-\mathbf{u})^{2}}{D R} f
$$

Substituting Eq. (2.4) into Eq. (2.1) results in 


$$
\frac{\partial g}{\partial t}+\mathbf{c} \frac{\partial g}{\partial \mathbf{x}}=-\frac{1}{\tau_{c}}\left(g-g^{e q}\right)+F_{g}+f q
$$

where

$$
\begin{gathered}
g^{e q}=\frac{(\mathbf{c}-\mathbf{u})^{2}}{D R} f^{e q} \\
F_{g}=\frac{(\mathbf{c}-\mathbf{u})^{2}}{2} F_{f}
\end{gathered}
$$

and

$$
q=\frac{\mathbf{c}-\mathbf{u}}{2}\left(\frac{\partial \mathbf{u}}{\partial t}+\mathbf{c} \cdot \nabla \mathbf{u}\right)
$$

Eq. (2.4) represents the internal energy carried by the particles and therefore Eq. (2.5) can be called as the evolution equation of internal energy density distribution function. The macroscopic variables can thus be redefined in term of distribution function $f$ and $g$ as

$$
\rho=\int f d \mathbf{c}, \rho \mathbf{u}=\int \mathbf{c} f d \mathbf{c}, \rho T=g d \mathbf{c}
$$

In this paper, we will apply the method proposed by Peng et al. [15] which consider that the viscous heat dissipation can be neglected for the incompressible flow. So here, we neglect the dissipation and the external force in the evolution equation of internal energy density distribution function as follow

$$
\frac{\partial g}{\partial t}+\mathbf{c} \frac{\partial g}{\partial \mathbf{x}}=-\frac{1}{\tau_{c}}\left(g-g^{e q}\right)
$$

By omitting the dissipation term, the complicated gradient operator in the evolution equation of internal energy distribution function can be dropped.

\section{Discretization Method}

In order to apply the lattice Boltzmann scheme into the digital computer, we need to discretised the evolution equation of the continuous lattice Boltzmann BGK equation for the momentum and energy. Expanding both of the equilibrium distribution function up to $\boldsymbol{u}^{2}$ results in [16]

$$
\begin{gathered}
f^{e q}=\rho\left(\frac{1}{2 \pi R T}\right)^{D / 2} \exp \left\{-\frac{\mathbf{c}^{2}}{2 R T}\right\}\left[1+\frac{\mathbf{c} \cdot \mathbf{u}}{R T}+\frac{(\mathbf{c} \cdot \mathbf{u})^{2}}{2(R T)^{2}}-\frac{\mathbf{u}^{2}}{2 R T}\right] \\
g^{e q}=\rho T\left(\frac{1}{2 \pi R T}\right)^{D / 2} \exp \left\{-\frac{\mathbf{c}^{2}}{2 R T}\right\}\left[1+\frac{\mathbf{c} \cdot \mathbf{u}}{R T}\right]
\end{gathered}
$$

Eq. (3.2) is obtained by assuming that at low Mach number flow (incompressible flow), the higher order of $\boldsymbol{u}^{2}$ and viscous heat dissipation can be neglected [15]. It also has been proved [17] that the above simplification does not alter the corresponding macroscopic equation of energy. The only change is the value of the constant parameter in the thermal conductivity which can be absorbed by manipulating the parameter $\tau_{\mathrm{c}}$.

To recover the macroscopic equation, the zeroth-to third-order moments of $f^{q q}$ and zeroth-to second-order moments of $g^{e q}$ must be exact. In general

$$
I_{f}=\int \mathbf{c}^{m} f^{e q} d \mathbf{c}, I_{g}=\int \mathbf{c}^{m} g^{e q} d \mathbf{c}
$$


where $I_{f}$ and $I_{g}$ should be exact for $m$ equal to zero till three and zero till two respectively. Now we introduce new variables $\phi_{f}$ and $\phi_{g}$ defined by

$$
\begin{aligned}
\phi_{f}(\mathbf{c})= & \mathbf{c}^{m} \rho\left(\frac{1}{2 \pi R T}\right)^{D / 2} \exp \left\{-\frac{\mathbf{c}^{2}}{2 R T}\right\}\left[1+\frac{\mathbf{c} \cdot \mathbf{u}}{R T}+\frac{(\mathbf{c} \cdot \mathbf{u})^{2}}{2(R T)^{2}}-\frac{\mathbf{u}^{2}}{2 R T}\right] \\
& \phi_{g}(\mathbf{c})=\mathbf{c}^{m} \rho T\left(\frac{1}{2 \pi R T}\right)^{D / 2} \exp \left\{-\frac{\mathbf{c}^{2}}{2 R T}\right\}\left[1+\frac{\mathbf{c} \cdot \mathbf{u}}{R T}\right]
\end{aligned}
$$

Rewriting Eq. (3.3) after substituting Eqs. (3.4) and (3.5) gives

$$
I_{f / g}(\mathbf{c})=\int \phi_{f / g}(\mathbf{c}) \exp \left\{-\frac{\mathbf{c}^{2}}{2 R T}\right\} d \mathbf{c}
$$

where $f$ and $g$ refer to the density and internal energy density distribution functions respectively.

Equation (3.6) can be calculated by using the Gauss-Hermite quadrature [18]. Hence, the Gauss-Hermite quadrature must consistently give accurate result for quadratures of zeroth-to-fifth-order of velocity moment for $f^{e q}$ and zeroth-to-third order for $g^{e q}$. This implies that we can choose third-order Gauss-Hermite quadrature in evaluating $I_{f}$ and second order Gauss-Hermitte quadrature for $I_{g}$. As a result, we obtained the expression for the discretised density equilibrium distribution function as follows

$$
f_{i}^{e q}=\rho \omega_{i}\left[1+3 \frac{\mathbf{c} \cdot \mathbf{u}}{c^{2}}+\frac{9(\mathbf{c} \cdot \mathbf{u})^{2}}{2 c^{4}}-\frac{3 \mathbf{u}^{2}}{2 c^{2}}\right]
$$

where $c=(3 R T)^{1 / 2}$ and the weights are $\omega_{1}=4 / 9, \omega_{2}=\omega_{3}=\omega_{4}=\omega_{5}=1 / 9$, and $\omega_{6}=\omega_{7}=\omega_{8}=\omega_{9}=1 / 36$. Lattice structure of this model is shown in Fig. 1.

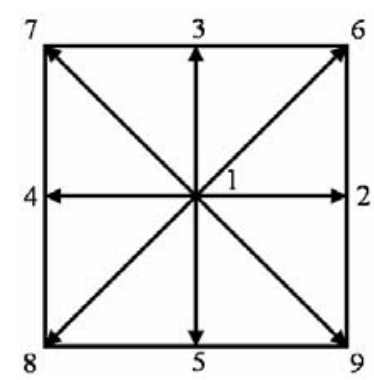

Figure 1. Lattice structure for 2-D density distribution function

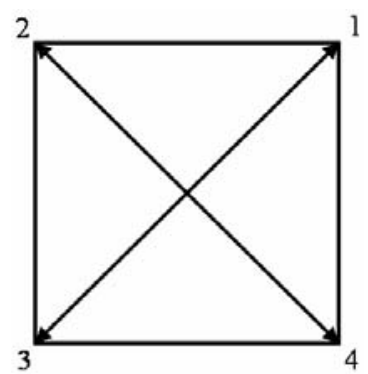

Figure 2. Lattice structure for 2-D internal energy density distribution function

After some modification in order to satisfy macroscopic energy equation via Chapmann-Enskog expansion procedure, the discretised internal energy density distribution function is obtained as

$$
g_{1,2,3,4}^{e q}=\frac{1}{4} \rho T\left[1+\frac{\mathbf{c} \cdot \mathbf{u}}{c^{2}}\right]
$$

This new type of lattice structure for internal energy density distribution is shown in Fig. 2.

While for three dimensional system, we obtained the equation for discretised density and internal energy density distribution function as below

$$
f_{i}^{e q}=\rho \omega_{i}\left[1+3 \frac{\mathbf{c} \cdot \mathbf{u}}{c^{2}}+\frac{9(\mathbf{c} \cdot \mathbf{u})^{2}}{2 c^{4}}-\frac{3 \mathbf{u}^{2}}{2 c^{2}}\right]
$$


with $\omega_{1}=8 / 27, \omega_{2 \sim 7}=2 / 27, \omega_{8 \sim 19}=1 / 54$ and $\omega_{20 \sim 27}=1 / 216$.

$$
g_{1 \sim 8}^{e q}=\frac{1}{8} \rho T\left[1+\frac{\mathbf{c} \cdot \mathbf{u}}{c^{2}}\right]
$$

respectively. The 3D lattice structures are shown in Figs. 3 and 4.

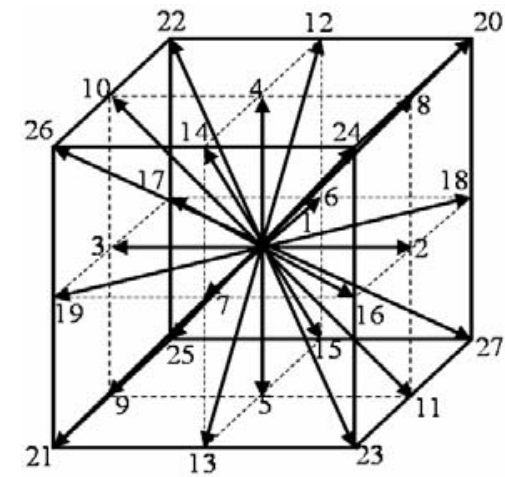

Figure 3. Lattice structure for 3-D density distribution function

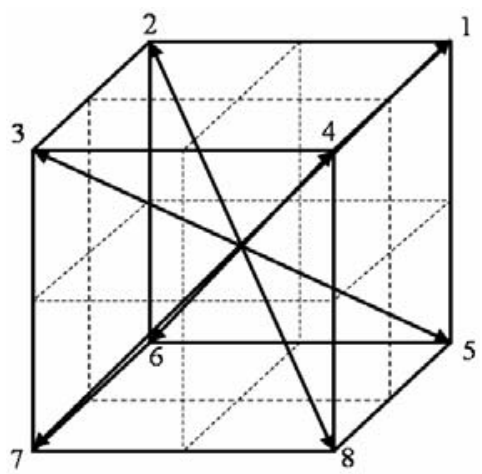

Figure 4. Lattice structure for 3-D internal energy density distribution function

Through a multiscaling expansion, the mass and momentum equations can be derived 2-D and 3-D models. The detail derivation is given by Luo et al. [19] and will not be shown here. The kinematic viscosity is given by

$$
v=\frac{2 \tau_{v}-1}{6}
$$

The energy equation at the macroscopic level can be expressed as follows

$$
\frac{\partial}{\partial t} \rho T+\nabla \cdot \rho \mathbf{u} T=\chi \nabla^{2}(\rho T)
$$

where $\chi$ is the thermal diffusivity. Thermal diffusivity and the relaxation time of internal energy distribution function is related as

$$
\chi=\tau_{c}-\frac{1}{2}
$$

\section{Numerical Simulations}

In the previous section, we have developed two types (2-D and 3-D) of lattice models for the internal energy density distribution function. In this section, we shall apply the newly developed models to simulate heat transfer in 2-D porous plate Couette flow problem and natural convection flow in a cubic cavity.

\section{Porous Plate Couette Flow}

Consider two infinite parallel flat plates separated by a distance of $L$. The upper cool plate at temperature $T_{c}$ moves at speed $U$, and the lower hot plate at temperature $T_{H}$ is stationary. A constant normal flow of fluid is injected through the bottom hot plate and withdrawn at the same rate from the upper plate. The analytical solution of the velocity field at steady state is given by 


$$
u=U\left(\frac{e^{\operatorname{Re}(y / L)}-1}{e^{\operatorname{Re}}-1}\right)
$$

where $R e$ is the Reynolds number based on the inject velocity $v_{0}$. The temperature profile in the steady state satisfies

$$
T=T_{C}+\Delta T\left(\frac{e^{\operatorname{Re} \operatorname{Pr}(y / L)}-1}{e^{\operatorname{RePr}}-1}\right)
$$

where $\Delta T=T_{H}-T_{C}$ is the temperature difference between the hot and cool walls. $\operatorname{Pr}=v / \chi$ is the Prandtl number. Another dimensionless parameter is the Rayleigh number defined by $R a=g \beta \Delta T L^{3} / v \chi$.

Periodic boundary conditions are used at the entrance and exit of the channel, and the non-equilibrium bounce back boundary conditions [20] for velocity. The unknown density distribution function at the boundary nodes can be determined from

$$
f_{\alpha}^{n e q}=f_{\beta}^{n e q}
$$

where $\mathbf{c}_{\alpha}$ and $\mathbf{c}_{\beta}$ have opposite directions.

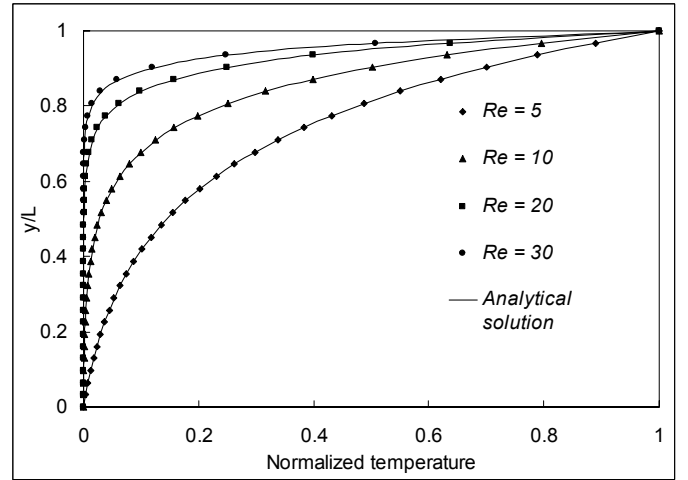

Figure 5. Temperature profile for $\operatorname{Pr}=0.71$ and $R a=100$

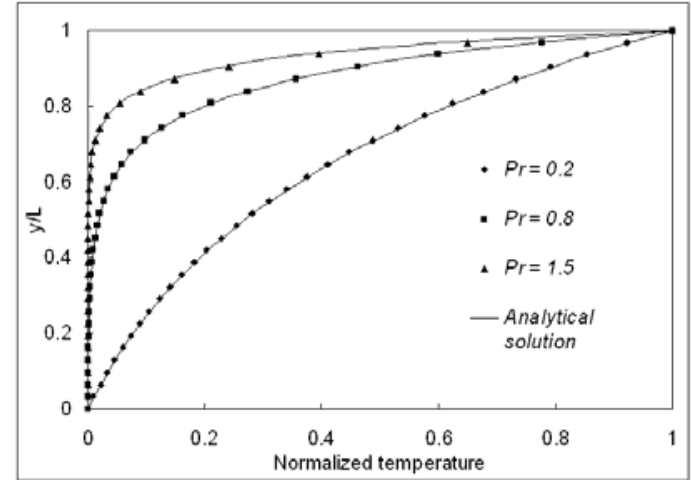

Figure 6. Temperature profile for $R a=100$ and $R e=10$

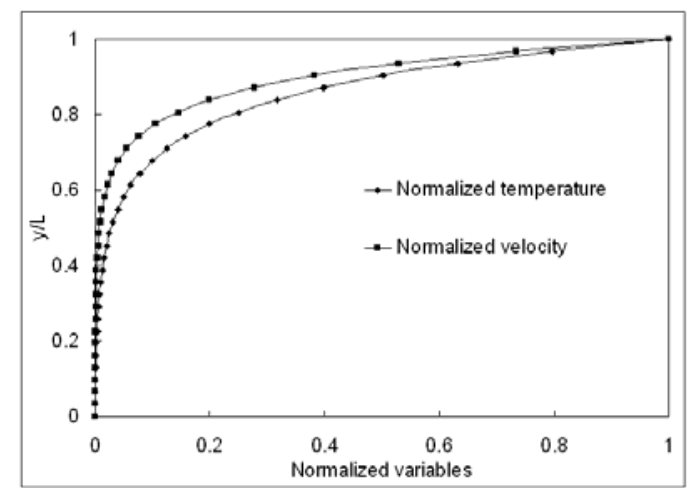

Figure 7. Velocity and temperature profile at $R e=10, P r=0.71$ and $R a=60000$

For temperature boundary condition, we used the non-equilibrium bounce back boundary condition proposed by Guo et al. [17]. For the known temperature at the boundary node $\mathrm{x}_{\mathrm{b}}$, the internal energy distribution function is given by 


$$
g_{i}\left(\mathbf{x}_{b}, t\right)=g_{t}^{e q}\left(\mathbf{x}_{b}, t\right)+g_{i}\left(\mathbf{x}_{f}, t\right)-g_{t}^{e q}\left(\mathbf{x}_{f}, t\right)
$$

where $x_{f}$ is the nearest fluid nodes.

The normalized temperature profile for $\operatorname{Pr}=0.71, R a=100$ and $R e=5,10,20$ and 30 is shown in Fig. 5. Fig. 6 shows the result for $R a=100, R e=10$ and $P r=0.2,0.8$ and 1.5. They agree well with the analytical solution. To show that our model is suitable and numerically stable for a wide range of Rayleigh number, we have done the computations for $R a=10$ till $R a=60000$ at $\operatorname{Pr}=0.71$ and $R e=10$. The result is shown in Fig. 7 .

\section{Natural Convection in a Cubic Cavity}

Numerical simulation for the natural convection flow in a cubic cavity was carried out to test the validity of the 3D eight-velocity thermal lattice Boltzmann model. Fig. 8 shows a schematic diagram of the setup in the simulation. No-slip boundary conditions [16] are imposed on all the faces of the cubes. The thermal conditions applied on the left and right wall are $T(x=0, y, z)=T_{H}$ and $T(x=1, y, z)=T_{C}$. The other faces being adiabatic, $\partial T / \partial n=0$, where $\partial T / \partial n$ is the appropriate normal derivative.

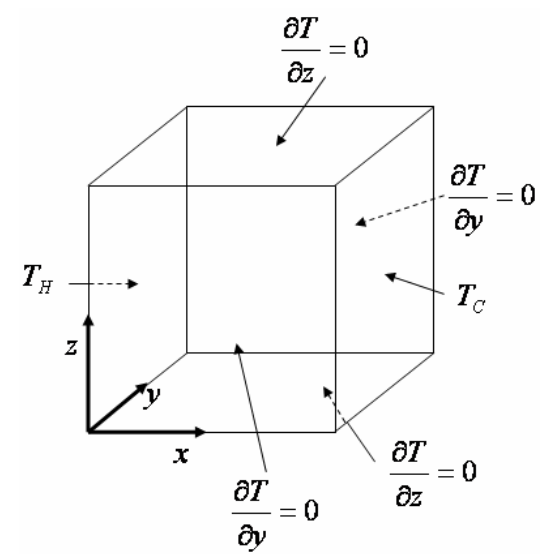

Figure 8 . Schematic geometry for natural convection in a cubic cavity

The temperature difference between the left and right walls introduces a temperature gradient in a fluid, and the consequent density difference induces a fluid motion, that is, convection. In the simulation, the Boussinesq approximation is applied to the buoyancy force term.

$$
\rho \mathbf{G}=\rho \beta g_{0}\left(T-T_{m}\right) \mathbf{j}
$$

where $\beta$ is the thermal expansion coefficient, $g_{0}$ is the acceleration due to gravity, $T_{m}$ is the average temperature and $\boldsymbol{j}$ is the vertical direction opposite to that of gravity. So the external force in Eq. (2.1) is

$$
F_{f}=3 \mathbf{G}(\mathbf{c}-\mathbf{u}) f^{e q}
$$

The dynamical similarity depends on two dimensionless parameters: the Prandtl number $P r$ and the Rayleigh number $R a$ defined as,

$$
\operatorname{Pr}=\frac{v}{\chi}, R a=\frac{g_{0} \beta \Delta T L^{3}}{v \chi}
$$

We carefully choose the characteristic speed $v_{c}=\left(g_{0} L T\right)^{1 / 2}$ so that the low-Mach-number approximation holds. The Nusselt number, $N u$ is one of the most important dimensionless numbers in describing the convective transport. The Nusselt number at the mid-plane is defined by 


$$
N u_{m p}=\int_{0}^{1} \frac{\partial T(y, z)}{\partial x} d z
$$

In all simulations, $\operatorname{Pr}$ is set to be 0.71 , and due to the limitation of computer capability, the grid sizes of $101 \times 101$ is used for $R a=10^{3}$ and $R a=10^{4}$. The grid dependence study has to be done before the comparison for $R a=10^{3}$. The result is given in Tab. 1. It can be clearly seen that as we increase the size of the mesh grid, the calculated variables converge to a constant value.

Table 1. Characteristic value, $R a=10^{3}$

\begin{tabular}{|c|c|c|c|c|c|}
\hline Grid & $61^{3}$ & $71^{3}$ & $81^{3}$ & $91^{3}$ & $101^{3}$ \\
\hline$u_{\max }$ & 3.564 & 3.533 & 3.556 & 3.553 & 3.553 \\
\hline$x$ & 0.52 & 0.51 & 0.51 & 0.52 & 0.52 \\
\hline$y$ & 0.82 & 0.81 & 0.81 & 0.82 & 0.82 \\
\hline$z$ & 0.50 & 0.50 & 0.50 & 0.50 & 0.50 \\
\hline$v_{\max }$ & 3.574 & 3.561 & 3.563 & 3.559 & 3.557 \\
\hline$x$ & 0.13 & 0.17 & 0.18 & 0.17 & 0.18 \\
\hline$y$ & 0.47 & 0.50 & 0.50 & 0.50 & 0.50 \\
\hline$z$ & 0.50 & 0.50 & 0.50 & 0.50 & 0.50 \\
\hline$w_{\max }$ & 0.174 & 0.173 & 0.173 & 0.173 & 0.173 \\
\hline$x$ & 0.50 & 0.50 & 0.50 & 0.50 & 0.50 \\
\hline$y$ & 0.50 & 0.50 & 0.50 & 0.50 & 0.50 \\
\hline$z$ & 0.25 & 0.24 & 0.25 & 0.24 & 0.25 \\
\hline$N u_{m p}$ & 1.093 & 1.095 & 1.094 & 1.094 & 1.094 \\
\hline
\end{tabular}
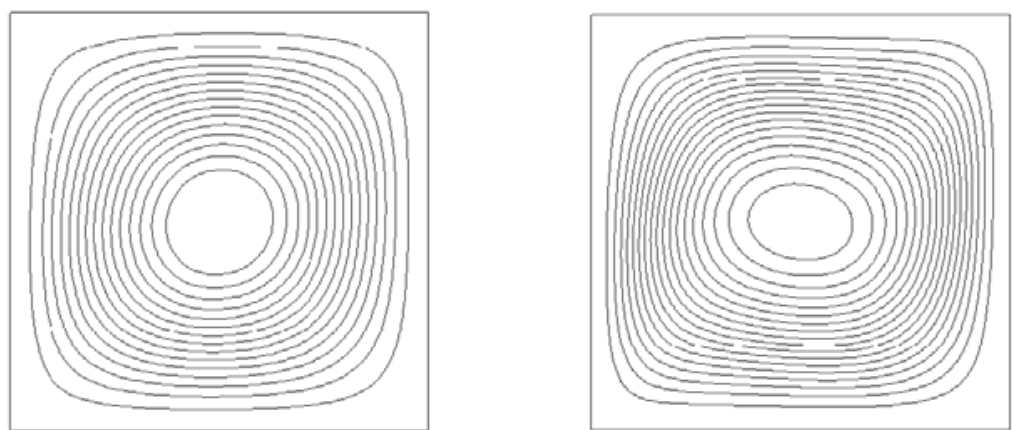

Figure 9. Streamlines of natural convection for $R a=10^{3}$ (left) and $R a=10^{4}$ (right)
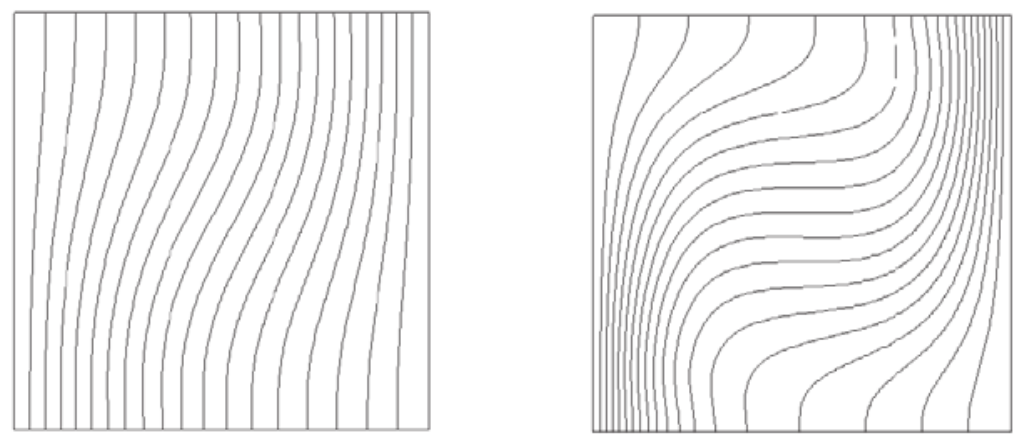

Figure 10. Isotherms of natural convection for $R a=10^{3}$ (left) and $R a=10^{4}$ (right)

Streamlines and isotherms predicted for flows at different Rayleigh numbers are shown in Figs. 9 and 10. At $R a=10^{3}$, streamlines are those of a single vortex, with its center in the center of the system. The corresponding 
isotherms are parallel to the heated walls, indicating that most of the heat transfer mechanism is by heat conduction. As the Rayleigh number increases, $\left(\mathrm{Ra}=10^{4}\right)$, the central streamline is distorted into an elliptic shape and the effects of convection can be seen in the isotherms.

For the simulation at $\mathrm{Ra}=10^{5}$, we used the same lattice type as in the density distribution function (27velocity model) for the internal energy density distribution function in order to avoid the stability problem reported by Azwadi [21]. The corresponding equilibrium internal energy density distribution function is given by

$$
g_{i}^{e q}=\rho T \omega_{i}\left[1+3 \frac{\mathbf{c} \cdot \mathbf{u}}{c^{2}}\right]
$$

where the weights are $\omega_{1}=8 / 27, \omega_{2 \sim 7}=2 / 27, \omega_{8 \sim 19}=1 / 54$ and $\omega_{20 \sim 27}=1 / 216$.

The numerical results for the simulation at $R a=10^{5}$ are shown in Fig. 11.
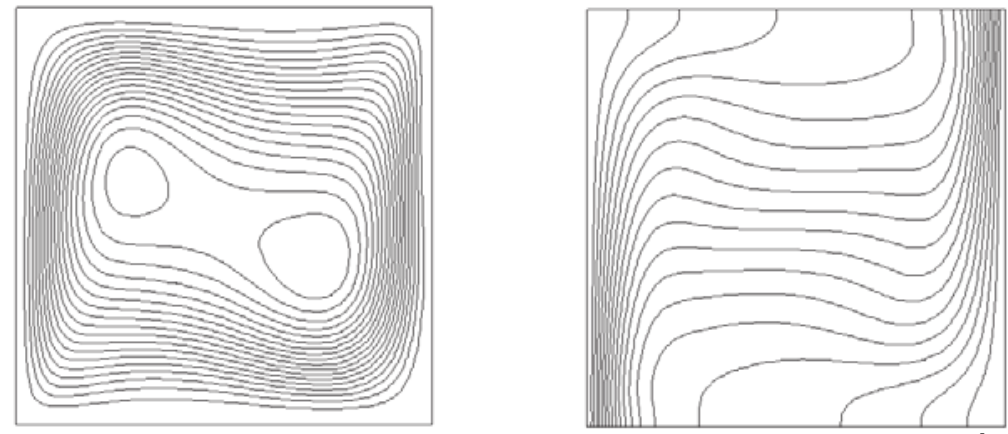

Figure 11. Streamlines (left) and isotherms (right) of natural convection for $R a=10^{5}$

Table 2. Comparison of numerical results between the present work and the "benchmark solutions" gathtered by Tric [22].

Table 2. Comparison of numerical results between the present work and the "benchmark solutions" gathtered by Tric [22].
\begin{tabular}{|c|c|c|c|c|c|c|}
\hline$R a$ & & \multicolumn{2}{|c|}{$10^{4}$} & \multicolumn{2}{c|}{$10^{5}$} \\
\hline Variables & Present work & Tric [22] & Present work & Tric [22] & Present work & Tric [22] \\
\hline$u_{\max }$ & 3.564 & 3.543 & 16.802 & 16.719 & 45.370 & 43.900 \\
\hline$x$ & 0.52 & 0.51 & 0.50 & 0.52 & 0.69 & 0.68 \\
\hline$y$ & 0.82 & 0.82 & 0.82 & 0.83 & 0.89 & 0.89 \\
\hline$z$ & 0.50 & 0.50 & 0.50 & 0.50 & 0.71 & 0.72 \\
\hline$v_{\max }$ & 3.557 & 3.544 & 19.279 & 18.983 & 73.080 & 71.060 \\
\hline$x$ & 0.82 & 0.82 & 0.90 & 0.93 & 0.93 & 0.93 \\
\hline$y$ & 0.50 & 0.50 & 0.52 & 0.52 & 0.49 & 0.50 \\
\hline$z$ & 0.50 & 0.50 & 0.73 & 0.73 & 0.89 & 0.88 \\
\hline$w_{\max }$ & 0.173 & 0.173 & 2.168 & 2.156 & 9.450 & 9.697 \\
\hline$x$ & 0.50 & 0.50 & 0.88 & 0.88 & 0.91 & 0.92 \\
\hline$y$ & 0.50 & 0.50 & 0.85 & 0.84 & 0.87 & 0.88 \\
\hline$z$ & 0.25 & 0.25 & 0.78 & 0.78 & 0.84 & 0.84 \\
\hline$N u_{m p}$ & 1.093 & 1.087 & 2.307 & 2.250 & 4.988 & 4.612 \\
\hline
\end{tabular}

At $R a=10^{5}$, the central streamline is elongated and two secondary vortices appear inside it. The isotherms become horizontal at the center of the cavity indicating that the dominant of heat transfer mechanism is convection.

A "benchmark solution" gathered by Tric [22] is brought for comparison and shown in Tab. 2. The table contains the numerical result of the maximum of each velocity component in the cavity, with its location and the Nusselt number at the mid-plane $(y=0.5)$. Note that the velocity shown in the table is normalized by the reference velocity of $\chi / H$, where $\chi$ is the thermal diffusivity and $H$ is is the height of the cavity.

From the results presented above, we can say that our new 3-D thermal lattice Boltzmann model has the capability to solve the thermal flow problems. 


\section{Conclusion}

In this paper, we have developed two types of lattice model which only use four (2-D) and eight (3-D) velocity components for internal energy density distribution function in incompressible limit. Both of the evolution equations have been directly derived from the continuous Boltzmann equation and Maxwell-Boltzmann equilibrium distribution function. We have demonstrated that by neglecting the compression work done by the pressure and viscous heat dissipation, this model does not include the complex gradient term in evolution equation and easier to be implemented as compared with the original thermal model.

The numerical result of the porous Couette flow problem for a wide range of Rayleigh numbers shows that our model is more suitable and numerically stable for the computational at high Rayleigh numbers. The numerical results also agree well with the analytical solutions.

Computations of natural convection in a cubic cavity correctly predicted the flow features for different Rayleigh numbers. The results are also in good agreement with those of previous studies. This shows that our model has the capability to solve the thermal flow problems.

\section{Acknowledgement}

The authors wish to thank Keio University, Universiti Teknologi Malaysia and the Malaysia Government for supporting this research.

\section{References}

[1] U. Frish, B. Hasslacher and Y. Pomeau, Phys. Rev. Lett., 56, 1505 (1986).

[2] S. Chen and G. Doolen, Annu. Rev. Fluid Mech., 30, 329 (1998).

[3] G. Breyiannis and D. Valougeorgis, Phys. Rev. E, 69, 065702 (2004).

[4] Halliday and C. M. Care, Phys. Rev. E, 53, 1602 (1996).

[5] L. Jonas, B. Chopard, S. Succi and F. Toschi, Phys. A, 362, 6 (2006).

[6] G. McNamara and B. Alder, Phys. A, 194, 218 (1993).

[7] H. Chen and C. Teixeira, Comp. Phys. Comm., 129, 21 (2000).

[8] C. Cercignani, The Boltzmann equations and its application, in Applied Mathematical Sciences (Springerverlag, New York, 1988).

[9] X. Shan, Phys. Rev. E, 55, 2780 (1997).

[10] L. S. Luo and X. He, Phys. Rev. E, 55, R6333 (1997).

[11] X. He, S. Shan and G. D. Doolen, J. Comp. Phys., 146, 282 (1998).

[12] P. L. Bhatnagar, E. P. Gross and M. Krook, Phys. Rev., 94, 511 (1954).

[13] S. Harris, An Introduction to the Theory of the Boltzmann Equation (Holt, Rinehart and Winston, New York, 1971).

[14] C. Cercignani, The Boltzmann equations and its application, in Applied Mathematical Sciences (Springer-Verlag, New York, 1988).

[15] Y. Peng, C. Shu and Y. T. Chew, Phys. Rev. E, 68, 020671 (2003).

[16] U. Frish, B. Hasslacher, D. Humieres, P. Lallemand, J. P. Rivet and Y. Pomeau, Complex Syst., 1, 649 (1987).

[17] Z. L. Guo, Y. Shi and T. S. Zhao, Phys. Rev. E, 70, 066310 (2004).

[18] P. J. Davis and P. Rabinowitz, Method of Numerical Integration (Academic Press, New York, 1984).

[19] L. S. Luo and X. He, J. Stats. Phys., 88, 927 (1997).

[20] Q. Zuo and X. He, Phys. Fluids, 9, 1591 (1997).

[21] C. S. Azwadi and T. Takahiko, Intl. J. Mod. Phys. B, 20, 2437 (2006).

[22] E. Tric, G. Labrosse and M. Betrouni, Int. J. Heat Mass Trans., 43, 4043 (2000). 